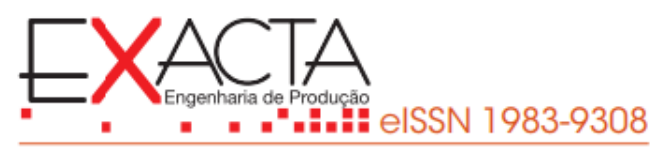

\title{
AVALIAÇÃO TÉCNICA-ECONÔMICA DO USO DA ENERGIA SOLAR FOTOVOLTAICA EM SUPERMERCADOS: UM ESTUDO DE CASO
}

\section{TECHNICAL-ECONOMIC EVALUATION OF THE USE OF SOLAR PHOTOVOLTAIC ENERGY IN SUPERMARKETS: A CASE STUDY}

Recebido em: 10 fev. 2021

Aprovado em: 29 ago. 2021

Versão do autor aceita publicada online: 29 ago. 2021

Publicado online: 03 set. 2021

\section{Como citar esse artigo - American Psychological Association (APA):}

Almeida Jr, M. dos. S., \& Altoé, L. (2022, out./dez.). Avaliação técnica-econômica do uso da energia solar fotovoltaica em supermercados: um estudo de caso. Exacta. 20(4), 1049-1061.

https://doi.org/10.5585/exactaep.2022.19371.

Submeta seu artigo para este periódico $\bigoplus$

Dados Crossmark 


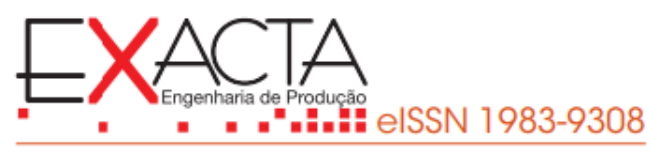

\title{
AVALIAÇÃO TÉCNICA-ECONÔMICA DO USO DA ENERGIA SOLAR FOTOVOLTAICA EM SUPERMERCADOS: UM ESTUDO DE CASO
}

\author{
TECHNICAL-ECONOMIC EVALUATION OF THE USE OF SOLAR PHOTOVOLTAIC \\ ENERGY IN SUPERMARKETS: A CASE STUDY
}

\author{
${ }^{1}$ Bacharel em Engenharia de Petróleo \\ Universidade Federal do Espírito Santo - UFES. \\ São Mateus, ES - Brasil. \\ marcio.ufes@gmail.com \\ ${ }^{2}$ Doutora em Engenharia Agrícola \\ Universidade Federal do Espírito Santo - UFES. \\ São Mateus, ES - Brasil. \\ leandra.altoe@ufes.br
}

Resumo: O uso de sistemas de geração distribuída tem sido uma alternativa procurada por gestores de estabelecimentos comerciais para reduzir custos com insumos energéticos. Objetivou-se com este trabalho analisar a viabilidade técnica-econômica da instalação de um sistema solar fotovoltaico em um supermercado, localizado no município de Jacinto - Minas Gerais. O sistema foi analisado utilizando o programa PVSyst e os indicadores Valor Presente Líquido (VPL), Taxa Interna de Retorno (TIR) e Tempo de Retorno de Capital (TRC). Encontrou-se VPL de R\$447.081,76, TIR de 24\% e TRC de 4 anos e 1 mês para a aquisição à vista do sistema; e VPL de R\$ 407.417,31, TIR de 19\% e TRC de 5 anos para aquisição por financiamento bancário. Portanto, o emprego de energia solar em supermercados tem o potencial de aumentar a competitividade destes estabelecimentos, além de contribuir para o desenvolvimento sustentável, cada vez mais exigido pelo mercado e pela sociedade.

Palavras-chave: Energia solar fotovoltaica. Viabilidade econômica e financeira. Setor comercial.

Abstract: The use of distributed generation systems has been an alternative sought by managers of commercial establishments to reduce costs with energy inputs. The objective of this work was to analyze the technical-economic feasibility of installing a photovoltaic solar system in a supermarket, located in the municipality of Jacinto - Minas Gerais. The system was analyzed using the PVSyst program and the indicators Net Present Value (VPL), Internal Rate of Return (TIR) and Capital Return Time (TRC). It was found VPL of $R \$ 447,081.76$, IRR of $24 \%$ and TRC of 4 years and 1 month for acquisition at sight of the system; and VPL of R\$407,417.31, TIR of $19 \%$ and TRC of 5 years for acquisition by bank financing. Therefore, the use of solar energy in supermarkets can increase the competitiveness of these establishments, in addition to contributing to sustainable development, which is increasingly demanded by the market and society.

Keywords: Photovoltaic solar energy. Economic and financial viability. Commercial sector. 
1 Introdução

O Brasil possui um amplo território com abundância em recursos naturais e, comparado ao panorama internacional, tem se destacado pelo uso de energia renovável, especialmente por fonte hidrelétrica (Lima et al., 2020; EPE, 2020). Porém, a dependência dos períodos de chuva e a não distribuição homogênea da mesma já comprometeu o suprimento nacional de energia (Dantas, Costa, \& Silva, 2016). Assim, para que o desenvolvimento econômico do país seja eficaz, será necessário produzir mais energia e por diferentes fontes nos próximos anos (Paim et al., 2019).

Há um grande potencial de energia solar existente no Brasil, uma vez que seu território recebe alta média de irradiação solar com grande proporção de dias ensolarados (Cardio, 2021; Drumond, Abel, \& Seabra, 2021). De acordo com Pereira et al. (2017), é possível gerar mais energia elétrica por sistemas fotovoltaicos no local menos ensolarado do Brasil do que no local mais ensolarado da Alemanha, a qual tem se destacado pelo uso desta tecnologia no cenário internacional. Em 2019, China, Estados Unidos, Japão, Alemanha e Índia, que lideravam o ranking mundial de energia solar, apresentavam uma capacidade fotovoltaica instalada de, respectivamente, 204,7 GW; 75,9 GW; 63 GW; 49,2 GW; e 42,8 GW (IEA, 2020), enquanto o Brasil possuía uma capacidade instalada de aproximadamente 2,0 GW para o mesmo ano (EPE, 2020).

Em 2012, foi promulgado um importante marco legal para promover o uso de fontes renováveis de energia no Brasil, a Resolução Normativa da Agência Nacional de Energia Elétrica n. 482 (ANEEL, 2012), atualizada pela Resolução Normativa n.o 687 (ANEEL, 2015) e pela Resolução Normativa n.o 786 (ANEEL, 2017a). Esse marco instituiu um sistema de compensação de energia elétrica no país, no qual unidades consumidoras com micro ou minigeração distribuída, a partir de fonte renovável ou cogeração qualificada, podem compensar seu consumo de energia. Por este sistema, realiza-se um balanço da energia injetada na rede e da energia consumida ao final do mês e, caso a produção de energia seja maior que o consumo, créditos são gerados para utilização em um período futuro.

Segundo Carstens e Cunha (2019), houve um crescimento da geração distribuída por energia solar fotovoltaica no Brasil a partir do ano da promulgação da Resolução Normativa ANEEL n.o 786/2012. Porém, analistas apontam a necessidade de novas melhorias no marco regulatório para difundir o aproveitamento desta fonte renovável em território nacional (Luna, Cunha, \& Torres, 2019). Além disso, outras barreiras existentes estão associadas ao custo e dificuldades dos investidores em financiar os sistemas fotovoltaicos, à pequena produção nacional de equipamentos fotovoltaicos e à logística onerosa e lenta (Rigo et al., 2019).

O setor comercial brasileiro é o terceiro maior consumidor de energia elétrica no Brasil, com participação de 17,4\%, atrás do setor industrial $(35,9 \%)$ e do setor residencial (26,1\%) (EPE, 2020). Quando se analisa especificamente o segmento de supermercados e hipermercados, as despesas com 
energia elétrica ocupam a segunda posição nos custos operacionais, perdendo apenas para a folha de pagamento de funcionários (ABRAS, 2017). Assim, é importante que os gestores busquem alternativas para reduzir as despesas com insumos energéticos e, consequentemente, promovam o aumento da competividade desses empreendimentos. Uma opção para isto é a geração distribuída de energia elétrica nos próprios supermercados e hipermercados, por exemplo, por meio de energia solar (Peroni, 2018).

Segundo estudo da Agência Nacional de Energia Elétrica (ANEEL, 2017b), o setor residencial é o que possui maior participação de consumidores com microgeração fotovoltaica no Brasil, com 79,5\%, seguido pelo setor comercial, com 15,0\% e dos setores industrial, rural e público, que juntos somam $5,5 \%$. Ainda de acordo com este estudo, sistemas fotovoltaicos destinados a atender os setores residencial e comercial apresentam, respectivamente, tempo de retorno de capital médio de 7 e 6 anos, considerando tarifas de diferentes concessionárias de energia elétrica atuantes no Brasil. Com base nestes dados, sistemas de energia fotovoltaica no setor comercial apresentam um tempo de retorno de capital médio mais curto do que no setor residencial, no entanto, a participação dos consumidores com microgeração fotovoltaica no setor comercial é menor do que no setor residencial no país.

Outros estudos têm indicado que a utilização de sistemas fotovoltaicos é economicamente viável no setor comercial brasileiro, comparado ao suprimento exclusivo pela rede de distribuição de energia elétrica. Entre eles, pode-se citar o trabalho de Peroni (2018), que avaliou o uso de energia fotovoltaica em supermercados localizados em diferentes municípios do estado de Minas Gerais e encontrou um tempo de retorno de capital médio de 4 anos e 8 meses, considerando um horizonte de planejamento de 25 anos. Paines, Vignochi, e Possamai (2018), por sua vez, analisaram a viabilidade da geração de energia fotovoltaica em edifícios comerciais no município de Florianópolis (SC) e encontraram um tempo de retorno de capital médio de 11 anos, com base em uma vida útil de 30 anos para o sistema fotovoltaico. Por fim, Romeral, Lima, e Dalto (2017) encontraram um tempo de retorno de capital de 14 anos para um sistema fotovoltaico destinado a atender um estabelecimento comercial no município de Londrina (PR), considerando um horizonte de planejamento de 25 anos. Portanto, os indicadores econômicos podem variar bastante a depender de parâmetros locais, como irradiação solar e tarifa de energia elétrica, sendo imprescindível realizar uma avaliação de viabilidade técnicaeconômica para auxiliar a tomada de decisão de empreendedores interessados em instalar projetos de energia fotovoltaica.

Neste contexto, este trabalho teve por objetivo realizar uma análise técnica-econômica para uso de energia solar fotovoltaica em um supermercado, localizado no município de Jacinto - Minas Gerais. Com isto, espera-se gerar informações que possam contribuir para as discussões sobre como a energia solar fotovoltaica pode potencializar a competitividade e aumentar a eficácia do setor varejista brasileiro. 


\section{Metodologia}

Para este estudo de caso, foi considerado um supermercado localizado no município de Jacinto, situado no Vale do Jequitinhonha, no estado de Minas Gerais. O estabelecimento é atendido pela concessionária de energia elétrica Companhia Energética de Minas Gerais (CEMIG), sendo enquadrado na classe comercial trifásico, subclasse B3, com tarifa única de demanda de potência e tarifas diferenciadas de consumo de energia elétrica de acordo com as horas de utilização do dia. Na Tabela 1 , está descrito o consumo de energia elétrica do estabelecimento no ano de 2018.

\section{Tabela 1}

Consumo de Energia Elétrica do Supermercado Estudado no Ano de 2018

\begin{tabular}{l|l}
\hline Mês & Consumo (kWh) \\
\hline Janeiro & 5.740 \\
\hline Fevereiro & 5.630 \\
\hline Março & 5.410 \\
\hline Abril & 5.255 \\
\hline Maio & 5.110 \\
\hline Junho & 5.005 \\
\hline Julho & 4.790 \\
\hline Agosto & 4.980 \\
\hline Setembro & 5.335 \\
\hline Outubro & 5.380 \\
\hline Novembro & 5.575 \\
\hline Dezembro & 5.710 \\
\hline Consumo mensal médio & 5.325 \\
\hline Consumo anual & 63.920 \\
\hline Sor & \\
\hline
\end{tabular}

Fonte: Elaborado pelos autores, com dados provenientes das faturas de energia elétrica do supermercado.

O dimensionamento e a análise técnica do sistema solar fotovoltaico foram realizados com auxílio do software Pvsyst, versão 6.88 (PVSyst, 2019), desenvolvido pela Universidade de Genebra na Suíça. A ferramenta computacional realiza as simulações considerando fatores geográficos e climáticos, o desempenho do sistema fotovoltaico, as perdas associadas à sombreamento e o coeficiente de reflexão, também conhecido como albedo, entre outros parâmetros. Neste estudo, foi considerado albedo com valor médio anual igual à 0,2 e que a área escolhida para instalação dos painéis solares não sofre sombreamento ao longo do dia. Além disso, o software Pvsyst possui uma extensa base de dados de painéis solares e inversores. Ao inserir os componentes do sistema fotovoltaico, o software verifica 
a compatibilidade dos dados de entrada de acordo com o datasheet dos equipamentos e, por fim, gera um relatório com informações técnicas sobre o sistema fotovoltaico.

O município de Jacinto (MG) se localiza a uma latitude de 16008'40" S e a uma longitude de 4017'36" O, está em uma altitude de 180 m em relação ao nível do mar, recebe irradiação horizontal global anual de $1.654,4 \mathrm{kWh} / \mathrm{m}^{2}$ e apresenta temperatura mensal média de $24,9^{\circ} \mathrm{C}$ (temperatura mínima de $22,6^{\circ}$ e temperatura máxima de $\left.26,7^{\circ}\right)$.

No dimensionamento, foi considerado que os painéis solares seriam instalados com $20^{\circ}$ de inclinação e $124^{\circ}$ de azimute. A Figura 1 apresenta dados de orientação e inclinação de painéis solares e a Figura 2 mostra dados de compatibilidade de equipamentos do sistema fotovoltaico inseridos no software Pvsyst para o caso estudado.

Figura 1

Captura de Tela do Software Pvsyst com Dados de Instalação dos Painéis Fotovoltaicos Para o Caso Estudado

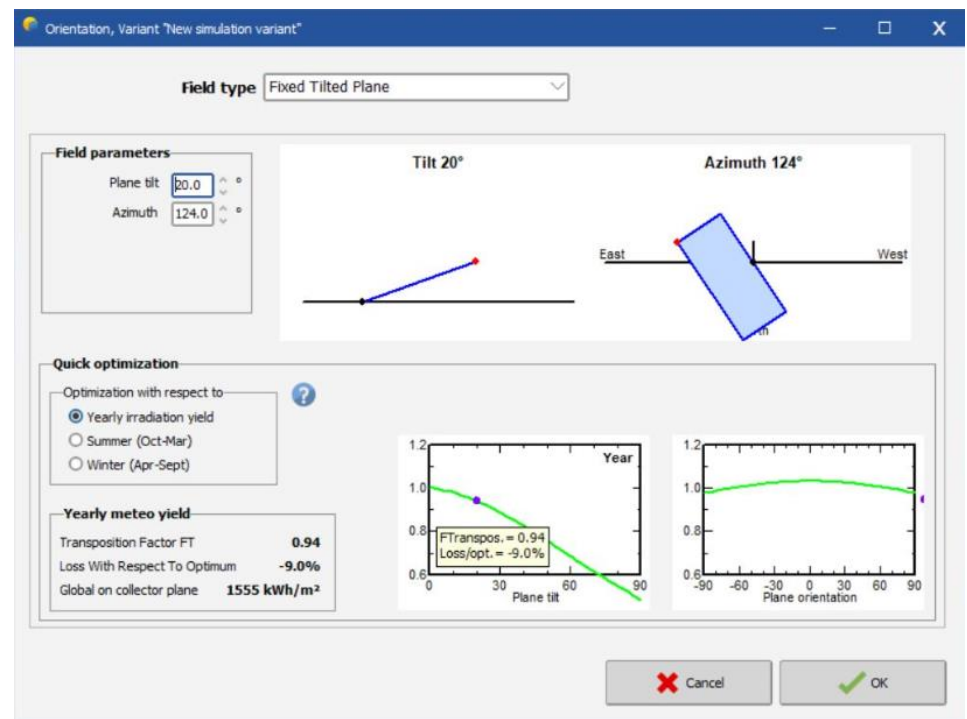

Fonte: Elaborado pelos autores. 


\section{Figura 2}

Captura de Tela do Software Pvsyst Com Seleção de Equipamentos do Sistema Fotovoltaico Para o Caso Estudado

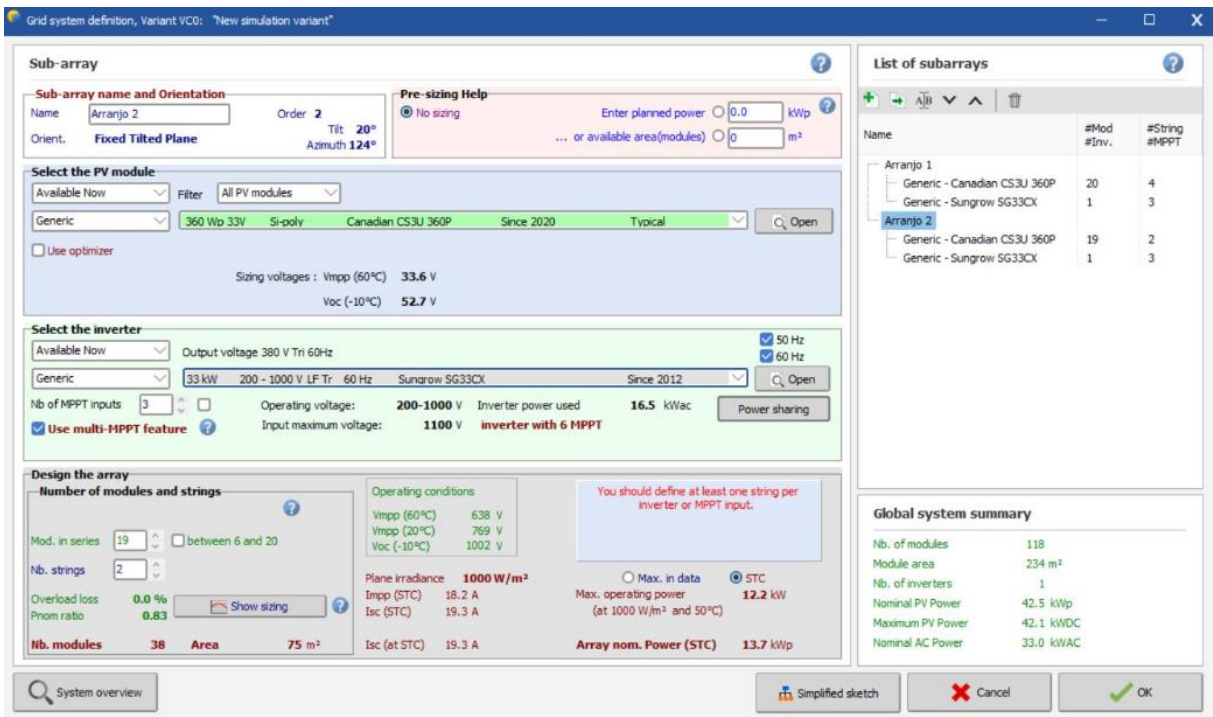

Fonte: Elaborado pelos autores.

Os parâmetros que são incluídos no cálculo da potência do sistema fotovoltaico consideram o consumo anual que o usuário necessita, descontado o custo de disponibilidade. Este custo refere-se a um valor cobrado pelas concessionárias para que as unidades com geração distribuída possam injetar o excedente de energia na rede e seu valor depende da tensão contratada pela unidade consumidora.

Para realizar a análise de viabilidade econômica do sistema solar fotovoltaico, comparado ao uso de energia elétrica da rede de distribuição, foram utilizados os seguintes indicadores: Valor Presente Líquido (VPL), Taxa Interna de Retorno (TIR) e Tempo de Retorno de Capital (TRC). Estes indicadores foram calculados para dois cenários hipotéticos: Cenário A (pagamento à vista do valor do sistema solar fotovoltaico) e Cenário B (pagamento por meio de financiamento bancário).

O Valor Presente Líquido refere-se ao fluxo de caixa final no horizonte de planejamento, subtraída a taxa de desconto, em moeda, com valor correspondente ao ano de 2019. O VPL foi calculado pela Eq. (1).

$$
V P L=\sum_{n=0}^{N} \frac{c_{n}}{(1+d)^{n}}
$$

Onde:

$$
\begin{array}{ll}
V P L & =\text { Valor Presente Líquido (R\$); } \\
C_{n} & =\text { Fluxo de caixa para o ano } \mathrm{n}(\mathrm{R} \$ / \mathrm{ano}) ; \\
n & =\text { O n-ésimo ano (adimensional); }
\end{array}
$$


$N=$ Horizonte de planejamento (anos); $\mathrm{e}$

$d \quad=$ Taxa de desconto (\% a.a).

A Taxa Interna de Retorno é a taxa de desconto que faz com que o Valor Presente Líquido seja igual a zero. A TIR foi calculada utilizando a Eq. (2).

$$
0=\sum_{n=0}^{N} \frac{C_{n}}{(1+T I R)^{n}}
$$

Onde:

$T I R=$ Taxa Interna de Retorno (\%);

$C_{n} \quad=$ Fluxo de caixa para o ano $\mathrm{n}(\mathrm{R} \$$ /ano);

$n=$ O n-ésimo ano (adimensional); $\mathrm{e}$

$N=$ Horizonte de planejamento (anos).

O Tempo de Retorno de Capital (TRC) refere-se ao número de anos necessários para que o fluxo de caixa líquido seja igual ao investimento inicial. A TRC foi calculada pela Eq. (3).

$$
T R C=\frac{C_{i}}{C_{\text {liq }}}
$$

Onde:

$$
\begin{array}{ll}
T R C & =\text { Tempo de Retorno de Capital (anos); } \\
C_{i} & =\text { Custo inicial (R\$); } \mathrm{e} \\
C_{\text {liq }} & =\text { Fluxo de caixa líquido anual esperado (R\$/ano). }
\end{array}
$$

O orçamento do sistema solar fotovoltaico foi fornecido por uma empresa especializada na data de 25 de outubro de 2020, considerando-se o valor real de mercado dos painéis solares, inversores, estrutura, necessidade de avaliação de um engenheiro elétrico, mão-de-obra para instalação do sistema, regulamentação burocrática junto a concessionária de energia elétrica, entre outros fatores. Dessa maneira, a seleção dos componentes do sistema solar fotovoltaico foi fundamentada na simulação técnica do software Pvsyst, possibilitando a associação do orçamento fornecido pela empresa, de acordo com os equipamentos indicados pela ferramenta computacional.

Para o cálculo dos indicadores econômicos, considerou-se uma taxa de desconto de 3,14\%, referente ao Índice Nacional de Preços ao Consumidor Amplo (IPCA) de 2019 e tarifa do kWh consumido junto a concessionária estadual para a classe comercial B3 de R\$0,88/kWh. Além disso, a análise de viabilidade econômica foi feita considerando um horizonte de planejamento de 25 anos, que é referente a garantia dos painéis do sistema solar fornecida pelo fabricante, e tem sido o tempo de vida útil de projetos fotovoltaicos adotado pela Agência Nacional e Energia Elétrica em análises econômicas (ANEEL, 2017b). Para o Cenário B, que diz respeito ao financiamento do sistema solar fotovoltaico, foi considerado o parcelamento do valor do investimento total, divido em 60 vezes com uma taxa de juros 
de 1,2\% ao mês, após consulta à uma instituição financeira privada com atuação no município de Jacinto (MG), que realiza financiamentos de projetos de energia solar.

\section{Resultados e discussão}

O estudo de caso analisado demandou um sistema solar fotovoltaico com potência de pico de 49,3 kW em condições normais de funcionamento. Na Tabela 2, é apresentado o dimensionamento do sistema, com o detalhamento dos equipamentos a serem utilizados, respeitando os parâmetros calculados e correlacionando-os com o datasheet do inversor e da potência dos painéis solares na aplicação do estudo de caso.

\section{Tabela 2}

Dimensionamento do Sistema Fotovoltaico Para o Supermercado Analisado

\begin{tabular}{l|l|l}
\hline Tipo & Produto & Quantidade \\
\hline Painéis solares & Canadian CS3W-360P & 137 \\
\hline Inversor & Sungrow SG33CX- 3-MPPT 380V & 1 \\
\hline Cabos & Cabo Solar 6 $\mathrm{mm}^{2}$ - Preto com proteção UV & $315 \mathrm{~m}$ \\
\hline Cabos & Cabo Solar 6 $\mathrm{mm}^{2}$ - Vermelho com proteção UV & $315 \mathrm{~m}$ \\
\hline Cabos & Cabo Solar 6mm² - Aterramento Verde com proteção UV & $315 \mathrm{~m}$ \\
\hline Transformador & Transformador Trifásico Isolador 380/220V 40kVa & 1 \\
\hline Monitoramento & Stick WIFI Sungrow & 1 \\
\hline Conectores & Staubli Conector - Kit PAR MC4 PV & 16
\end{tabular}

Fonte: Elaborado pelos autores.

O sistema fotovoltaico não seria suficiente para zerar a conta de energia elétrica do supermercado, devido a alguns fatores, como não considerar o consumo do horário de pico, taxas fixadas pela classificação do grupo B3, além da cobrança pelo uso da rede de distribuição. Porém, os resultados são satisfatórios no que diz respeito a geração e abatimento na conta de energia elétrica ao longo do ano. A área necessária para instalação dos painéis solares foi de $272 \mathrm{~m}^{2}$, os quais podem ser dispostos na cobertura do supermercado ou do estacionamento de veículos.

O projeto prevê a instalação de 137 painéis solares divididos em dois arranjos, sendo o primeiro arranjo composto por 80 módulos, com quatro strings (sequência de módulos conectados em série) em paralelo, onde cada string contém 20 módulos em série; e o segundo arranjo composto por três strings em paralelo, com conexão de 19 módulos em série. Dessa forma, é possível garantir a viabilidade técnica conforme a compatibilidade do datasheet dos equipamentos do sistema. A Figura 3 mostra os dados da 
geração de energia elétrica do sistema solar fotovoltaico e o consumo de energia elétrica do supermercado, previsto para suprir a demanda energética durante 12 meses.

Figura 3

Geração de Energia do Sistema Fotovoltaico e Consumo de Energia Elétrica do Supermercado, ao Longo de 12 Meses

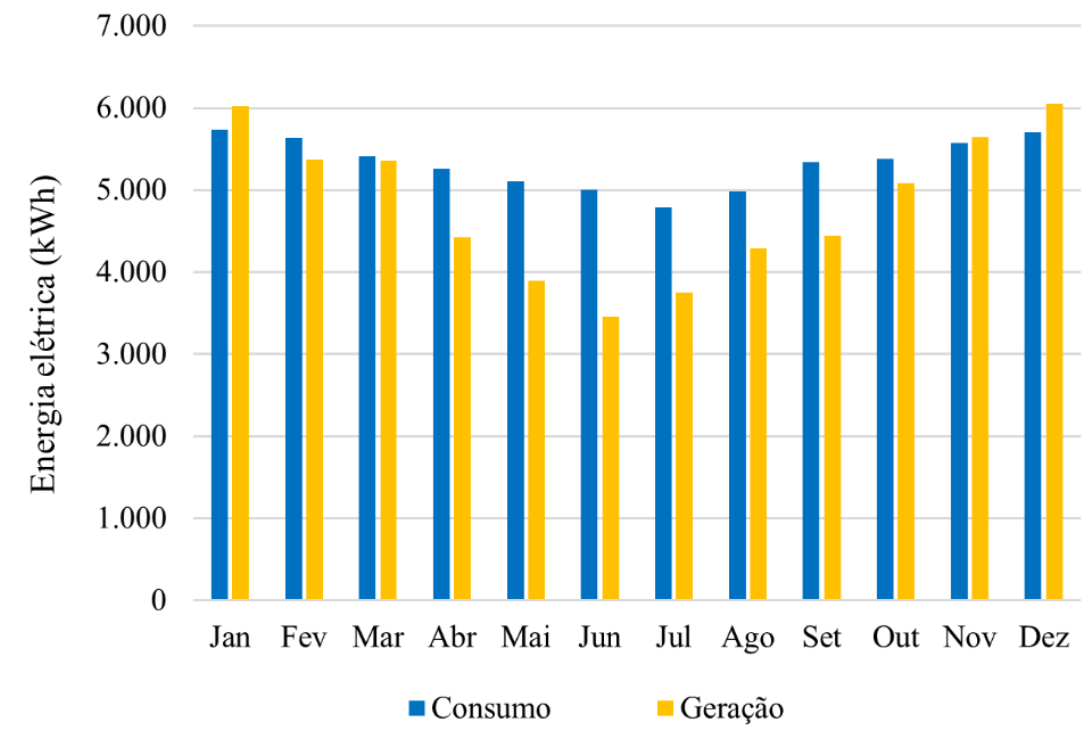

Fonte: Elaborado pelos autores.

Os resultados da análise de viabilidade econômica do sistema solar fotovoltaico para o caso estudado são apresentados na Tabela 3. Ressalta-se que o Cenário A diz respeito ao pagamento à vista e o Cenário B ao pagamento via financiamento bancário do sistema fotovoltaico.

\section{Tabela 3}

Indicadores de Viabilidade Econômica do Sistema Fotovoltaico Para o Supermercado Analisado

\begin{tabular}{l|l|l}
\hline Parâmetros & Cenário A & Cenário B \\
\hline Investimento & $\mathrm{R} \$ 138.667,00$ & $\mathrm{R} \$ 168.000,00$ \\
\hline Valor Presente Líquido & $\mathrm{R} \$ 447.081,76$ & $\mathrm{R} \$ 407.417,31$ \\
\hline Taxa Interna de Retorno & $24 \%$ & $19 \%$ \\
\hline Taxa de Retorno de Capital & 4 anos e 1 mês & 5 anos \\
\hline
\end{tabular}

Fonte: Elaborado pelos autores. 
O custo de aquisição do sistema fotovoltaico (Investimento) do Cenário A foi de 17,5\% inferior

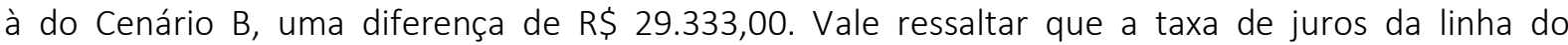
financiamento tem forte influência na composição do custo total e, consequentemente, nos indicadores de viabilidade econômica. Assim, recomenda-se que os interessados em instalar projetos de energia fotovoltaica busquem por diferentes instituições financeiras para encontrar a melhor linha de financiamento para seu perfil consumidor.

O Valor Presente Líquido do projeto de energia solar fotovoltaico para ambos os cenários foi expressivo, em que o VPL do Cenário A foi de 9,7\% superior à do Cenário $B$, uma diferença de $R$ \$ 39.664,45. O custo inicial do Cenário B é zero, pois o investimento será diluído ao longo do tempo do financiamento de 5 anos, com uma parcela de $R \$ 2.800,00$ ao mês, valor este que é próximo à fatura de energia elétrica paga pelo supermercado. Portanto, para o estudo de caso, mesmo que o empreendedor não tivesse o capital para a compra à vista, seria viável a aquisição do sistema solar fotovoltaico via financiamento bancário, mantendo-se o custo com suprimento de energia elétrica próximo ao atual.

O Valor Presente Líquido é um dos indicadores mais utilizados em análises de viabilidade econômica, sendo possível obter uma perspectiva real do valor do dinheiro no futuro (Campos, 2019). Este indicador representa a soma das entradas e saídas de um fluxo de caixa no dia inicial do projeto (Castanheira, 2016). Ao analisar projetos de investimento de capital, o cálculo da TIR pode ser muito útil como segundo critério, por indicar até que taxa de custo de capital os projetos analisados são favoráveis. Em resumo, considera-se as seguintes situações: o custo capital $<T I R$, indica que o projeto deve ser aceito (e VPL >0); o custo capital = TIR, torna-se indiferente aceitar ou não (VPL = 0); e o custo capital > $T I R$, indica que o projeto deve ser recusado (VPL $<0)$.

Dessa forma, a Taxa Interna de Retorno é aceita em ambos os cenários de acordo com a taxa de desconto considerada, neste caso, igual à 3,14\%. Para um projeto ser viável, a Taxa Interna de Retorno deve ser maior que a taxa mínima de atratividade e quanto maior a TIR, melhor rentabilidade. A Taxa Interna de Retorno do Cenário A foi de cinco pontos percentuais superior à do Cenário B, uma diferença de $26,3 \%$ entre os dois casos.

A análise do Tempo de Retorno de Capital refere-se ao tempo necessário para que o capital investido se torne zero, ou seja, é o tempo em que o projeto devolve o capital investido. Ao contrário da TIR, quanto menor o TRC mais rentável o projeto, pois mais rápido as receitas serão convertidas em lucro (Villamarim, 2016). O Tempo de Retorno de Capital do Cenário A foi de 18,3\% inferior à do Cenário $B$, uma diferença de 11 meses.

Outros estudos na área têm demonstrado a viabilidade econômica de implementação de sistemas solares fotovoltaicos no setor comercial brasileiro e que os indicadores, como Valor Presente Líquido, Taxa Interna de Retorno e Tempo de Retorno de Capital, dependem de vários fatores, entre 
eles, investimento inicial, nível de irradiação solar no local, eficiência dos equipamentos do sistema fotovoltaico e condições de tributação de energia elétrica (ANEEL, 2017b; Romeral, Lima, \& Dalto, 2017; Peroni, 2018; Ramos et al., 2021).

\section{Considerações finais}

O presente estudo teve como finalidade realizar uma análise técnica-econômica para implantação de energia solar fotovoltaica em um supermercado localizado na cidade de Jacinto (MG). O software que é comumente utilizado por empresas do setor elétrico para o dimensionamento de sistemas fotovoltaicos permite simulações que consideram diversos detalhes, como fatores climáticos, orientação e inclinação dos painéis solares, influência das perdas globais do sistema, entre outros, buscando aproximar ao máximo a aplicação do projeto à realidade do local.

A instalação do sistema solar fotovoltaico para este estudo de caso mostrou ser economicamente viável, comparado ao uso de energia elétrica exclusivamente pela rede de distribuição, apresentando melhores indicadores para o cenário de compra à vista, comparado ao de financiamento bancário. Obteve-se VPL de R\$447.081,76, TIR de 24\% e TRC de 4 anos e 1 mês para a aquisição à vista do sistema; e VPL de R\$ 407.417,31, TIR de 19\% e TRC de 5 anos para aquisição por financiamento bancário. Assim, o Valor Presente Líquido e a Taxa Interna de Retorno foram de, respectivamente, 9,7\% e 26,3\% superiores e o Tempo de Retorno de Capital foi de 18,3\% inferior para o cenário à vista em comparação ao cenário de financiamento. Todavia, ressalta-se que a decisão do melhor cenário deve ser tomada pelo investidor com base nas condições financeiras do empreendimento. Para os dois casos há níveis adequados quanto à viabilidade econômica e, consequentemente, potencial para reduzir os custos financeiros com energia elétrica do supermercado.

Com base nos resultados deste estudo e de outros trabalhos na área, verifica-se que os indicadores de viabilidade econômica de sistemas solares fotovoltaicos podem variar, uma vez que dependem fortemente de fatores locais, com destaque para disponibilidade de irradiação solar e preço da tarifa de energia elétrica. Assim, ao planejar substituir o suprimento de energia da rede de distribuição, total ou parcialmente, por geração solar fotovoltaica, é imprescindível realizar um estudo de viabilidade técnica-econômica para cada caso específico.

Por fim, o emprego de energia solar em supermercados tem o potencial de aumentar a competitividade destes estabelecimentos, além de contribuir para o desenvolvimento sustentável, cada vez mais exigido pelo mercado e pela sociedade. 


\section{Referências}

ABRAS (2017). Acordo entre ENGIE e ACATS promete diminuir os gastos do setor com energia. Disponível: https://www.abras.com.br/clipping/noticias-abras/60254/acordo-entre-engie-eacats-promete-diminuir-os-gastos-do-setor-com-energia.

ANEEL (2012). Resolução Normativa n.o 482, de 17 de abril de 2012. Estabelece as condições gerais para o acesso de microgeração e minigeração distribuída aos sistemas de distribuição de energia elétrica, o sistema de compensação de energia elétrica, e dá outras providências. Brasília, DF: Diário Oficial da União.

ANEEL (2015). Resolução Normativa n. o 687, de 24 de novembro de 2015. Altera a Resolução Normativa no 482, de 17 de abril de 2012, e os Módulos 1 e 3 dos Procedimentos de Distribuição - PRODIST. Brasília, DF: Diário Oficial da União.

ANEEL (2017a). Resolução Normativa no 786, de 17 de outubro de 2017. Altera a Resolução Normativa no 482, de17 de abril de 2012. Brasília, DF: Diário Oficial da União.

ANEEL (2017b). Nota Técnica $n^{\circ}$ 0056/2017-SRD/ANEEL. Atualização das projeções de consumidores residenciais e comerciais com microgeração solar fotovoltaicos no horizonte 2017-2024. Brasília, DF: Agência Nacional de Energia Elétrica.

Campos, L. C. (2019). Análise da atratividade de sistemas fotovoltaicos no mercado de micro geração distribuída em municípios de pequeno e médio portes, via indicadores socioeconômicos. Estudo de caso: Município de Itajubá (Dissertação de Mestrado). Universidade Federal de Itajubá, Itajubá, MG. Disponível: https://repositorio.unifei.edu.br/xmlui/handle/123456789/1966.

Cardio, L. G. T. (2021). Mitigating the risk of photovoltaic power generation: A complementarity model of solar irradiation in diverse regions applied to Brazil. Utilities Policy, 71, 101245. DOI: https://doi.org/10.1016/j.jup.2021.101245.

Carstens, D. D. S., \& Cunha, S. K. (2019). Challenges and opportunities for the growth of solar photovoltaic energy in Brazil. Energy Policy, 125, 396-404. DOI: https://doi.org/10.1016/j.enpol.2018.10.063.

Castanheira, N. P. (2016). Cálculo aplicado à gestão e negócios (1a ed.) Curitiba: Intersaberes.

Dantas; F. C., Costa, E. M., \& Silva, \& J. L. M. (2016). Elasticidade preço e renda da demanda por energia elétrica nas regiões brasileiras: uma abordagem através de painel dinâmico. Revista de Economia, 43 (3), 1-20. DOI: http://dx.doi.org/10.5380/re.v42i3.36594.

Drumond Jr, P., Castro, R. D., \& Seabra, J. A. E. (2021) Impact of tax and tariff incentives on the economic viability of residential photovoltaic systems connected to energy distribution network in Brazil. Solar Energy, 224, 462-47. DOI: https://doi.org/10.1016/j.solener.2021.06.034.

EPE (2020). Balanço Energético Nacional 2020: ano base 2019. Disponível: https://www.epe.gov.br/pt/publicacoes-dados-abertos/publicacoes/balanco-energeticonacional-2020.

IEA (2020). Report IEA-PVPS T1-37:202: snapshot of global PV markets 2020 Disponível: https://ieapvps.org/wp-content/uploads/2020/04/IEA_PVPS_Snapshot_2020.pdf. 
Lima, M. A., Mendes, L. F. R., Mothé, G. A., Linhares, F. G., Castro, M. P. P., Silva, M. G., \& Sthel, M. S. (2020). Renewable energy in reducing greenhouse gas emissions: Reaching the goals of the Paris agreement in Brazil. Environmental Development, 33, 100504. DOI: https://doi.org/10.1016/j.envdev.2020.100504.

Luna, M. A. R., Cunha, F. B., \& Torres, E. A. (2019). Solar Photovoltaic Distributed Generation in Brazil: The Case of Resolution 482/2012. Energy Procedia, 159, 484-490. DOI: https://doi.org/10.1016/j.egypro.2018.12.036.

Paim, M. A., Dalmarco, A. R., Yang, C. H., Salas, P., Lindner, S., Mercure, J. F., Guerra, J. B. S. O. A., Derani, C., Silva, T. B., \& Viñuales, J. E (2019). Evaluating regulatory strategies for mitigating hydrological risk in Brazil through diversification of its electricity mix. Energy Policy, 128, 393401. DOI: https://doi.org/10.1016/j.enpol.2018.12.064.

Paines, P. A., Vignochi, L., \& Possamai, O. (2018). Simulação de sistema fotovoltaico para o setor comercial. Exacta, 16 (3), 17-30. DOI: https://doi.org/10.5585/exactaep.v16n3.7579.

Pereira, E. B., Martins, F. R., Gonçalves, A. G., Costa, R. S., Lima, F. J. L., Rüther, R., Abreu, S. L., Tiepolo, G. M., Pereira, S. V., \& Souza., J. G (2017). Atlas brasileiro de energia solar (2a ed). São José dos Campos: INPE. Disponível: http://labren.ccst.inpe.br/atlas_2017.html.

Peroni, M. B. (2018). Viabilidade econômico-financeira e barreiras para o avanço da energia solar fotovoltaica no setor de supermercados (Tese de Doutorado). Fundação Getúlio Vargas, Rio de Janeiro, RJ. Disponível: http://bibliotecadigital.fgv.br/dspace/handle/10438/24123.

PVSyst (2019). Software PVSyst V 6.88. Disponível: https://www.pvsyst.com/download-pvsyst.

Ramos, L. C., Oliveira, G. G., Gouvêa, M. T., Amaral, M. A., Guimarães, H. P., \& Rosa, M. T. M. G. (2021). Análise da viabilidade e dimensionamento de placas fotovoltaicas para uma microempresa do setor de alimentos. Brazilian Journal of Development, 7 (8), 29593-29614. DOI: https://doi.org/10.34117/bjdv7n3-599.

Rigo, P. D., Siluk, J. C. M., Lacerda, D. P, Rosa, C. B., \& Redisk, G. (2019) Is the success of small-scale photovoltaic solar energy generation achievable in Brazil? Journal of Cleaner Production, 240, 118243. DOI: https://doi.org/10.1016/j.jclepro.2019.118243.

Romeral, P. A. A. F., Lima, M. T., Dalto, J. L. (2017) Análise de viabilidade econômico-financeira para a implementação de um sistema fotovoltaico em uma distribuidora de peças automotivas na cidade de Londrina - PR. Revista Produção Industrial \& Serviços, 4 (2), 77-90. Disponível: https://periodicos.uem.br/ojs/index.php/rev_prod/article/view/52379.

Villamarim, J. A. (2016). Análise da viabilidade técnica-econômica de um sistema eólico-fotovoltaico com conexão à rede elétrica suprindo uma estação rádio base de telefonia celular no estado de São Paulo-SP (Dissertação de Mestrado). Universidade de São Paulo, SP. Disponível: https://teses.usp.br/teses/disponiveis/3/3143/tde-23012017-150207/pt-br.php. 